OPEN ACCESS

Edited by:

Ivan Rychlik,

Veterinary Research Institute,

Czech Republic

Reviewed by: Haider Abdul-Lateef Mousa,

University of Basrah, Iraq

Chang H. Kim

Purdue University College

of Veterinary Medicine, USA

*Correspondence:

Rustam I. Aminov

rustam.aminov@gmail.com

Specialty section:

This article was submitted to

Infectious Diseases,

a section of the journal

Frontiers in Microbiology

Received: 02 March 2016 Accepted: 09 May 2016

Published: 24 May 2016

Citation:

Ktsoyan ZA, Mkrtchyan MS, Zakharyan MK, Mnatsakanyan AA, Arakelova KA, Gevorgyan ZU, Sedrakyan AM, Hovhannisyan Al, Arakelyan AA and Aminov RI (2016) Systemic Concentrations of Short Chain Fatty Acids Are Elevated in Salmonellosis and Exacerbation of Familial Mediterranean Fever.

Front. Microbiol. 7:776

doi: 10.3389/fmicb.2016.00776

\section{Systemic Concentrations of Short Chain Fatty Acids Are Elevated in Salmonellosis and Exacerbation of Familial Mediterranean Fever}

\author{
Zhanna A. Ktsoyan', Mkhitar S. Mkrtchyan', Magdalina K. Zakharyan', \\ Armine A. Mnatsakanyan ${ }^{2}$, Karine A. Arakelova', Zaruhi U. Gevorgyan ${ }^{2}$, \\ Anahit M. Sedrakyan', Alvard I. Hovhannisyan ${ }^{1}$, Arsen A. Arakelyan ${ }^{1}$ and \\ Rustam I. Aminov ${ }^{3 *}$
}

'Institute of Molecular Biology of National Academy of Sciences of Republic of Armenia, Yerevan, Armenia, ${ }^{2}$ Clinical Hospital of Infectious Diseases Nork, Ministry of Health of Republic of Armenia, Yerevan, Armenia, ${ }^{3}$ School of Medicine and Dentistry, University of Aberdeen, Aberdeen, UK

Gut microbiota-produced short chain fatty acids (SCFAs) play an important role in the normal human metabolism and physiology. Although the gradients of SCFAs from the large intestine, where they are largely produced, to the peripheral blood as well as the main routes of SCFA metabolism by different organs are known well for the healthy state, there is a paucity of information regarding how these are affected in disease. In particular, how the inflammation caused by infection or autoinflammatory disease affect the concentration of SCFAs in the peripheral venous blood. In this work, we revealed that diseases caused either by infectious agents (two Salmonella enterica serovars, S. Enteritidis, and S. Typhimurium) or by the exacerbation of an autoinflammatory disease, familial Mediterranean fever (FMF), both result in a significantly elevated systemic concentration of SCFAs. In the case of salmonellosis the concentration of SCFAs in peripheral blood was significantly and consistently higher, from 5- to 20-fold, compared to control. In the case of FMF, however, a significant increase of SCFAs in the peripheral venous blood was detected only in the acute phase of the disease, with a lesser impact in remission. It seems counterintuitive that the dysbiotic conditions, with a reduced number of gut microorganisms, produce such an effect. This phenomenon, however, must be appraised within the context of how the inflammatory diseases affect the normal physiology. We discuss a number of factors that may contribute to the "leak" and persistence of gut-produced SCFAs into the systemic circulation in infectious and autoinflammatory diseases.

Keywords: Salmonella, salmonellosis, short chain fatty acids, inflammation, familial Mediterranean fever, peripheral blood

\section{INTRODUCTION}

Gut microbiota plays a fundamental role in the development and maintenance of many functions of the human body throughout life. The interactions with the host involve a number of metabolic, signaling, developmental, and immune processes. It is also important for the maintenance of integrity of the gut ecosystem and also for the protection against the invading pathogens 
(colonization resistance; Nicholson et al., 2012) via microbial and immune mechanisms (Stecher et al., 2010). Alterations in the composition and metabolic activities of the gut microbiota contribute to many chronic and degenerative diseases (Hawrelak and Myers, 2004). The interplay between the environmental factors such as antibiotic usage, diet, stress, infections and injury as well as the host genetic factors continuously shape the structure-and-function of the intestinal microbiota, with the consequences for human health. A number of dysbiotic conditions are associated with a wide spectrum of human diseases such as inflammatory bowel disease (IBD), colorectal cancer, irritable bowel syndrome (IBS), stomach ulcers and cancer, asthma, atopy, metabolic syndrome, diabetes, hypertension, autism, and other pathologies (Penders et al., 2007; Prakash et al., 2011; Petersen and Round, 2014; Parekh et al., 2015).

The interplay between the gut microbiota and immune system is complex. Although, it is well-established that the gut microbiota is essential for the proper development and maintenance of the immune system (Cerf-Bensussan and Gaboriau-Routhiau, 2010), there are indications that the immune system, on its turn, may also affect the composition of the gut microbiota (Khachatryan et al., 2008; Manukyan et al., 2008b). This interaction may have important implications for the development of inflammatory diseases, including autoimmune diseases and allergy, and the mechanisms by which the gut commensals drive the development of different types of immune responses are beginning to be understood (Licciardi et al., 2010).

Inflammation is the integral part of the normal innate immune response and acts as an early step in the cascade of reactions eventually resulting in the resolution of inflammation and healing (Headland and Norling, 2015). The sequence of events from the induction to resolution of inflammation in the course of infection of bacterial, viral, or protozoal etiology is fairly wellknown. Nevertheless, some details of this process, in particular how the innate inflammatory response affects specific pathogens, commensal microbiota and the metabolome are less understood.

Regarding the inflammatory response, it becomes clear that it is not always detrimental to the pathogen. Some pathogens, for example, may use it for their own advantage during infection (Stecher et al., 2010). Activating the host immune defenses can suppress the protective commensal microbiota and facilitate the invasion and establishment of pathogens. Salmonella-induced inflammation, for example, results in the altered microbiota, which favors the growth of the pathogen (Barman et al., 2008). The ability of Salmonella spp. to overcome colonization resistance and successfully invade the host cells is partially due to its ability to utilize the inflammation-induced ecological and metabolic breaches to enhance its pathogenic potential (Winter et al., 2010; Ahmer and Gunn, 2011).

The normal intestinal microbiota generates high concentrations of short chain fatty acids (SCFAs) as the end products of anaerobic metabolism in the gut. The SCFAs produced execute multiple effects on the host as well as on the microbiota itself. Some are partially metabolized by the colonocytes (Velázquez et al., 1997) while the remaining SCFAs are cleared in the liver and then enter systemic circulation (Schuijt et al., 2012). The gradient of concentrations of the three main SCFAs (acetate, propionate, and butyrate) in the human body has been initially studied by Cummings et al. (1987). They found that the highest concentration of these three SCFAs is in the large intestine, with a gradual decrease in the portal, hepatic and peripheral venous blood. The concentration in peripheral blood was $1 / 1000$ th of that in the large intestine. The SCFAs delivered to various tissues then affect lipid, glucose, and cholesterol metabolism (den Besten et al., 2013). For example, SCFAs have a profound effect on the appetite regulation and energy homeostasis of the host (Byrne et al., 2015). They also contribute to the maintenance of immune homeostasis in the intestine and have multiple effects on the host cells involved in the inflammatory and immune pathways (Honda and Littman, 2012). In particular, SCFAs have a profound effect on regulation of $\mathrm{T}$ cells and contribute, directly or indirectly, to their differentiation (Kim et al., 2014). Other SCFAs effects on host physiology and metabolism include the regulation of epithelial cell growth and contribution to the functioning of the central and peripheral nervous system (Bienenstock et al., 2015).

Although it is known that SCFAs can modulate the function of immune cells, more studies are necessary in order to understand the precise role of specific gut bacterial SCFAs on host immune cells in vivo (Vinolo et al., 2011). The previous studies have been essentially limited to one of the main SCFAs produced, butyrate, and the experimental basis has been limited to animal models, in vitro experiments, and some clinical intervention studies. There are very few in vivo studies aimed at clarification of the SCFAs role in disease. For example, it has been demonstrated that individual SCFAs or their combination may enhance disease resistance and modulate the expression of an antimicrobial host defense peptide (Sunkara et al., 2011, 2012). High systemic concentrations of propionate and butyrate, however, are toxic and may impose adverse effects on the host (Bloemen et al., 2010). Still, as has been stated in a recent review by den Besten et al. (2013): "A coherent understanding of the multilevel network in which SCFAs exert their effects is hampered by the lack of quantitative data on actual fluxes of SCFAs and metabolic processes regulated by SCFAs."

On the microbial side, the effects of SCFAs are mainly metabolic, supporting a number of syntrophic relationships in the gut (Pimentel et al., 2012). At the same time, in some pathological inflammatory conditions such as infection these substances can be used by a pathogen to enhance its virulence potential for the invasion of the intestinal epithelium of the host (Winter et al., 2010).

In our previous works we have established that in diseases involving an inflammatory component the concentrations and profiles of gut microbial products in systemic circulation, in particular that of long chain fatty acids, are substantially different compared to control (Ktsoyan et al., 2011, 2013a). The main aim of the present study was to establish how the quantity and proportion of the SCFAs, which are generated by the commensal microbiota and then enter systemic circulation, are affected in two diseases. The first disease investigated included infections by two Salmonella enterica serovars, and the second - an autoinflammatory disease, familial Mediterranean fever (FMF; Brydges and Kastner, 2006; Manukyan and Aminov, 
2016). In both conditions we observed the increase of systemic concentrations of SCFAs as well as changes in the molar ratio of main SCFAs.

\section{MATERIALS AND METHODS}

In this study, which was performed over a 2 -years period from 2013 to 2015, we examined 64 Armenians residing in the territory of Republic of Armenia. Five cohorts were investigated: (i) control group of healthy subjects $(n=18)$; (ii) patients with acute salmonellosis caused by $S$. Typhimurium $(n=11)$ or (iii) S. Enteritidis $(n=15)$ infections; (iv) FMF patients with acute inflammation $(n=9)$; and (v) FMF patients in remission $(n=11)$.

All patients and controls belonged to the same ethnic group. The FMF cases were diagnosed based on Tel-Hashomer criteria with further genetic confirmation of $M E F V$ mutations (Livneh et al., 1997). None of the FMF patients and healthy individuals used antibiotics within 3 months prior to sampling.

The cohorts of salmonellosis patients included subjects admitted to the infectious disease hospital Nork in Yerevan, RA. Diagnoses were based on clinical presentations and laboratory analyses. Clinical appearances consistent with gastroenteritis were diarrhea, fever, nausea, vomiting, and abdominal cramps. Anamnesis included food and water consumed, social gatherings, relatives and friends with the similar symptoms, and the history of any recent travel.

The salmonellosis patient selection criteria for this study included not taking any type of medication including antibiotics before the hospital admission. Blood and fecal samples were taken on the first or the second day of hospital admission. At the time of discharge from the hospital, no Salmonella presence was detected in the feces or blood of the patients.

Biochemical tests for the identification of Salmonella were fermentation of glucose, negative urease reaction, lysine decarboxylase, negative indole test, $\mathrm{H}_{2} \mathrm{~S}$ production, and fermentation of galactitol (dulcitol). Serotypes of Salmonella were determined using the standard Kauffman-White scheme with the use of commercially available polyvalent antisera for flagellar $(\mathrm{H})$ and lipopolysaccharide $(\mathrm{O})$ antigens.

All subjects in the study, or their parent or guardian if a child, were informed about the aim of this study and gave their written consent to participate in it. The study protocol was approved by the Ethics Committee of the Institute of Molecular Biology NAS RA (IORG number 0003427, Assurance number FWA00015042, and IRB number 00004079).

Quantitative and qualitative determination of SCFAs in the peripheral blood of patients was performed by gas chromatography (GC) using Varian GC CP-3380 (Varian, Inc., USA) and WCOT Fused Silica type columns (length - $50 \mathrm{~m}$ and inner diameter $-0.32 \mathrm{~mm}$ ). The blood samples were taken by venipuncture in the amount of $5 \mathrm{ml}$. After the clot formation, a sample was centrifuged and $\mathrm{pH}$ of $1 \mathrm{ml}$ of supernatant was adjusted to 2.0 with $50 \%$ sulfuric acid. The extraction with $1 \mathrm{ml}$ of diethyl ether was performed twice and then evaporated down to $100 \mu \mathrm{l}$. A portion of the resulting ether extract $(1 \mu \mathrm{l})$ was subjected to GC analysis. Identification of SCFAs was performed by determining the relative retention times; commercially available analytical grade SCFAs were used as standards. Quantification of SCFAs was done by comparing the height of peaks to the standard solutions with known concentrations. Before each series of tests the steady state of chromatograph operation was achieved, followed by calibration with standards.

GraphPad Prism 5 (GraphPad Software, USA) was used to perform the Mann-Whitney $U$-test to determine the statistical significance of differences among the groups studied. The $p$-values $<0.05$ were considered statistically significant. Discriminant function analysis was carried out with the IBM SPSS Statistics 19 package (IBM, USA).

\section{RESULTS}

\section{Systemic SCFAs in Salmonellosis Patients}

The concentration of SCFAs in the peripheral blood of healthy subjects was found to be consistently low (Figures $\mathbf{1}$ and $\mathbf{3}$ ). This is not surprising since it is well-known that these microbial metabolites are actively metabolized, both in situ in the gastrointestinal tract (GIT) and in other organs such as the liver, muscles and other organs and tissues. On the contrary, the concentration of SCFAs in the peripheral blood of patients with acute salmonellosis caused by $S$. Enteritidis and $S$. Typhimurium infections was significantly and consistently higher compared to control (from 5- to 20-fold, $p<0.05$; Figure 1). The only exception was the concentration of caproate, which was significantly increased only in patients infected by $S$. Enteritidis, but not by $S$. Typhimurium, compared to control. Between the two serovars, significant differences $(p<0.05)$ were detected in the concentration of isovalerate (higher in S. Typhimurium) and caproate (higher in $S$. Enteritidis; Figure 1).

The SCFA concentration data were further subjected to multivariate statistic analyses, namely discriminant function analyses (DA; Figure 2). The predictive accuracy of model based on the SCFA concentration variables was sufficiently accurate at $94.7 \%$, with only two $S$. Enteritidis infection cases being allocated to the control group (Figure 2). These results suggested that despite the similarity in the increase of SCFAs concentrations in response to both $S$. enterica infections, there is certain specificity in the corresponding SCFA profile, which depends on the serotype of the pathogen. We have found earlier that the inflammatory response in salmonellosis is serotypespecific (Ktsoyan et al., 2013b, 2015). The differential immune response probably results in the differential composition of the gut microbiota, with the subsequent distinctive profile of SCFAs excreted.

\section{Systemic SCFAs in Autoinflammatory Disease FMF}

Systemic concentration of the majority of SCFAs in FMF patients during disease exacerbation was consistently higher compared 


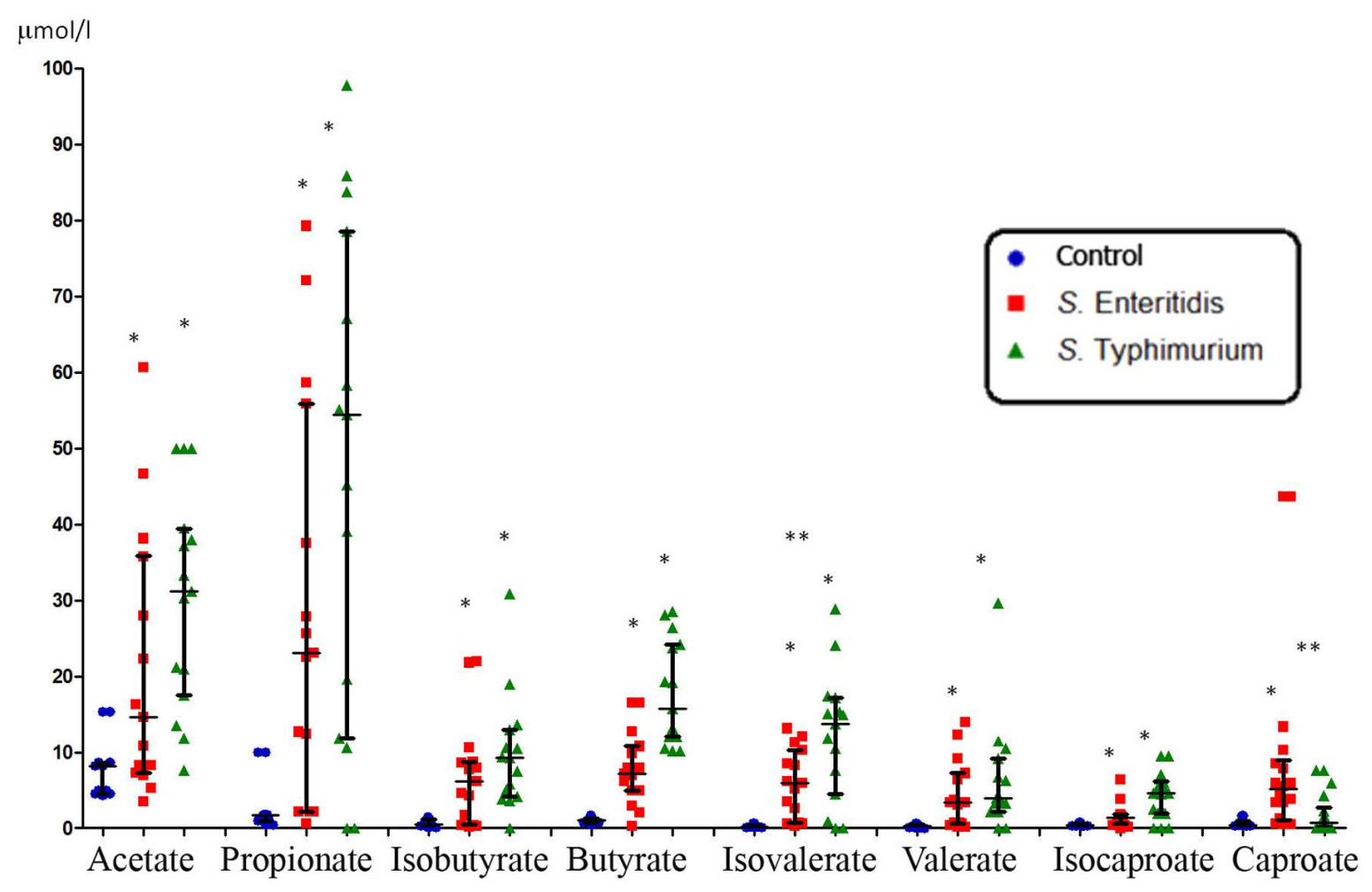

FIGURE 1 | Systemic concentration of SCFAs in patients infected with two serotypes of Salmonella enterica (median with interquartile range). Statistically significant differences in SCFA concentrations: * significant difference between infected and control subjects ( $p \leq 0.05$, Mann-Whitney $U$-test) and ** significant difference between $S$. Enteritidis- and $S$. Typhimurium-infected patients ( $p \leq 0.05$, Mann-Whitney $U$-test).

to control (Figure 3). Statistically significant differences were detected for almost all SCFAs, except valerate. In remission, however, the levels of SCFAs were much lower compared to the attack period and were closer to the values in the control group, except acetate (Figure 3). Comparison between the disease stages revealed that the concentration of the majority of SCFAs was significantly higher during the attack period compared to remission except for valerate (Figure 3).

Consistently with these observations, our further DA analysis of the three groups revealed a closer clustering of the control and FMF remission groups compared to the disease exacerbation group (Figure 4). In general, however, the model had a good predictive power, with an overall $94.1 \%$ accuracy of classifications. Thus, despite that the systemic concentration of the majority of SCFAs is not significantly different between the FMF remission and control groups, there is still a specific profile of SCFAs in disease remission allowing to separate this group from control. We have shown earlier that although FMF remission is characterized by the absence of clinical signs of disease, the elevated level of cytokines and CRP suggest the persisting subclinical inflammation (Manukyan et al., 2008a). Thus the subclinical inflammation during the remission periods may affect the composition of gut microbiota and, subsequently, the specific profile of SCFAs excreted.

Thus the results demonstrated that the systemic concentration of microbially produced SCFAs in acute FMF is significantly elevated compared to control. The concentration of the majority of SCFAs in FMF remission is not significantly increased compared to control but, nevertheless, displays a SCFAs profile different from that of control.

\section{Ratio of the Main Systemic SCFAs}

Finally, we determined the molar ratio of the main SCFAs in peripheral venous blood (Table 1). The three most abundant SCFAs generated by gut microbiota are acetate, propionate and butyrate, together comprising more than $95 \%$ of all SCFAs (Cook and Sellin, 1998), and which are present in a molar ratio of about 60:20:20 in the large intestine (Cummings et al., 1987). In the peripheral blood these three SCFAs also remain dominant. In our experiments we also found that these SCFAs are dominant in the venous blood of all patients and controls (Figures 1 and 3). The molar ratio of acetate:propionate:butyrate in healthy subjects was approximately 80:10:10 (Table 1) suggesting the preferential utilization of the two latter SCFAs. In salmonellosis the proportions of butyrate and propionate increased up to 15 and 50\%, correspondingly, at the expense of acetate, which proportion fell dramatically to $35 \%$ (Table 1). On the contrary, the proportion of systemic acetate in FMF is even higher compared to control (90 vs. $80 \%$ ), with the proportional decrease of the percentage of propionate and butyrate, both falling to $5 \%$ (Table 1). 


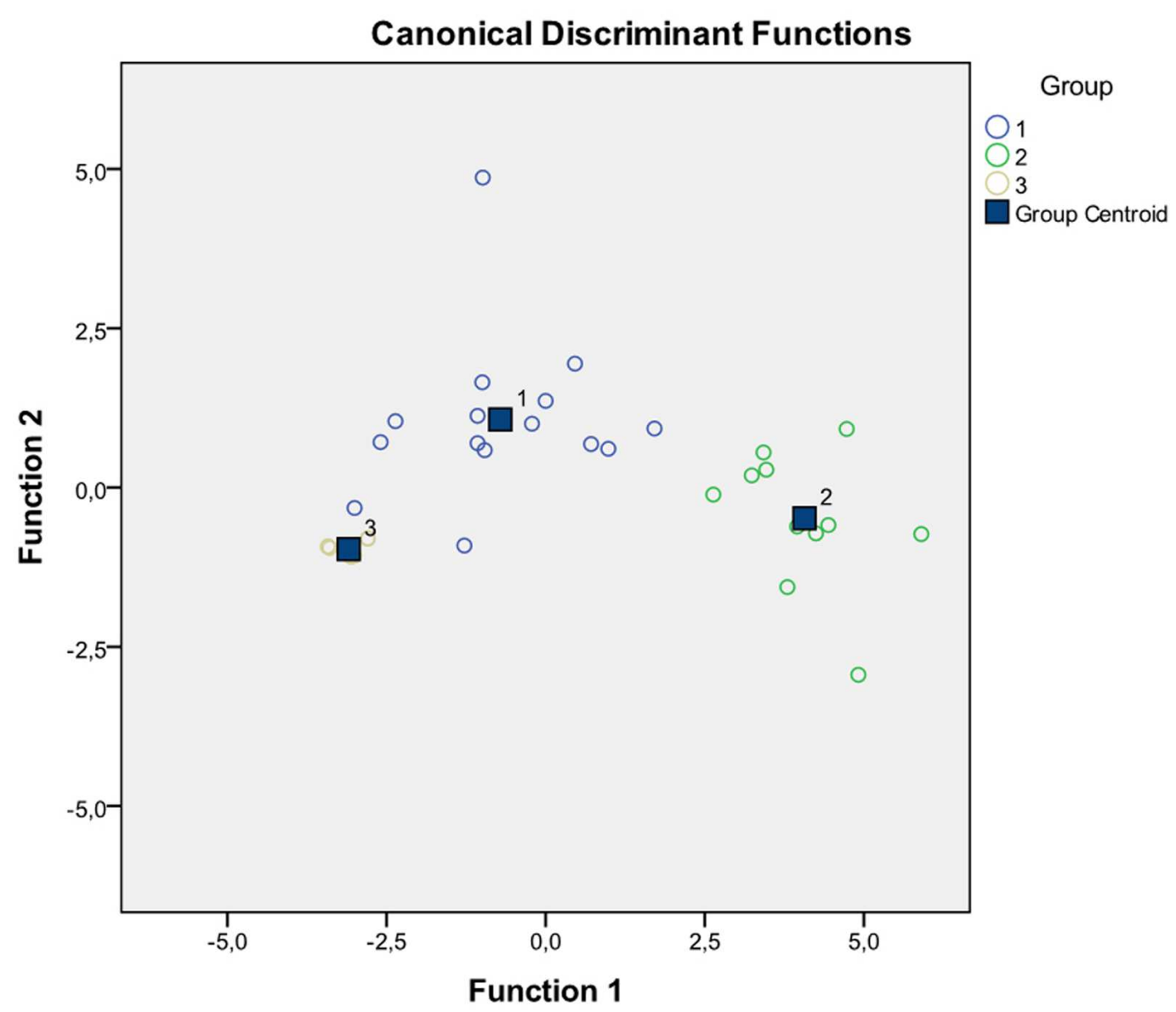

\begin{tabular}{|l|c|c|c|c|}
\hline \multirow{2}{*}{ Cohorts } & \multicolumn{3}{|c|}{ Predicted group membership } & Total, \\
\cline { 2 - 4 } & SE, N (\%) & ST, N (\%) & Control, N (\%) & N (\%) \\
\hline 1. SE & $13(86.7)$ & $0(0)$ & $2(13.3)$ & $15(100)$ \\
\hline 2. ST & $0(0)$ & $11(100)$ & $0(0)$ & $11(100)$ \\
\hline 3. Control & $0(0)$ & $0(0)$ & $11(100)$ & $11(100)$ \\
\hline
\end{tabular}

FIGURE 2 | Scatterplot of DA model and the predicted group membership in the model based on systemic concentration of microbial SCFAs in salmonellosis patients and control subjects. Number of variables in the model -8 and grouping consists of three groups. Root $1,2-$ discriminant functions 1 and 2 (1st and 2nd canonical roots). Cohorts: (1) S. Enteritidis-infected patients (SE), (2) S. Typhimurium-infected patients (ST), (3) control subjects. Predictive accuracy of classification: $94.7 \%$ of the original group cases were correctly classified; Wilks' $\lambda=0.055$.

\section{DISCUSSION}

In this work, we established that two types of diseases with an inflammatory component, of the infectious and autoinflammatory nature, result in the significantly elevated systemic levels of microbially produced SCFAs. In the autoinflammatory disease, FMF, this increase is mainly associated with the acute inflammation stage but even in remission the profile of SCFAs remains biased compared to control. To the best of our knowledge this is the first attempt to measure systemic concentrations of SCFAs in pathological conditions such as a gastrointestinal infection or an autoinflammatory disease.

Microbial anaerobic metabolism of dietary and endogenous substrates in the human gut results in the production of 


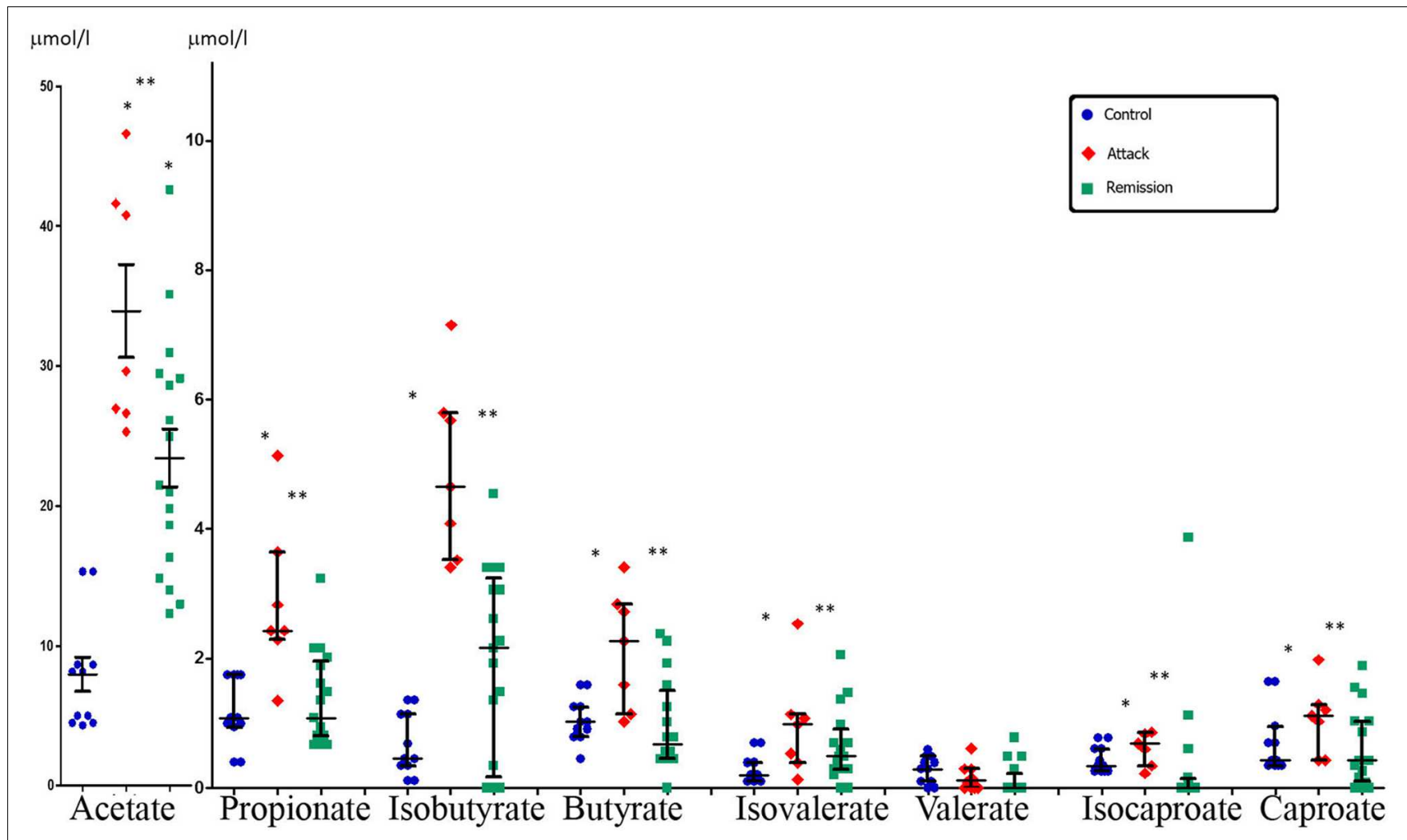

FIGURE 3 | Systemic concentration of SCFAs in FMF patients in attack and remission periods and in control subjects (median with interquartile range). Statistically significant differences in SCFA concentrations: * significant difference between FMF attack and control ( $p \leq 0.05$, Mann-Whitney $U$-test) and ** significant difference between FMF attack and remission ( $\rho \leq 0.05$, Mann-Whitney $U$-test). The concentration of acetate is shown in a different scale.

SCFAs and gasses such as hydrogen, methane, and carbon dioxide. A pioneering effort to establish the fate of the three main microbially produced SCFAs (i.e., acetate, propionate, and butyrate) has been made by Cummings et al. (1987) using the autopsy material of sudden death victims. They determined the SCFAs concentration in different parts of the large intestine and in portal, hepatic and peripheral venous blood and found that the concentration in peripheral blood was $1 / 1000$ th of that in the intestine. They also noticed a marked difference in the molar ratio of these SCFAs in the three parts of circulation. Our data in regards to the concentration of propionate and butyrate in the peripheral blood of healthy volunteers are consistent with these earlier findings. The concentration of acetate, however, is substantially lower possibly reflecting other confounding factors. Besides colonic fermentation, acetate can be produced endogenously (Skutches et al., 1979; Scheppach et al., 1991). The level of acetate is also affected by the host metabolism since its involvement in cholesterol biosynthesis and lipidogenesis, as a cosubstrate for glutamine and glutamate synthesis, as well as serving as energy source for many organs and tissues (Knowles et al., 1974; Wong et al., 2006; den Besten et al., 2013). A substantially lower concentration of acetate in peripheral venous blood compared to that obtained by Cummings et al. (1987) has been also reported by others (Dankert et al., 1981). The molar ratio of acetate to propionate to butyrate concentrations in the peripheral blood of healthy subjects, $80: 10: 10$, is within the range of the previously published data (Cummings et al., 1987; Peters et al., 1992; Bloemen et al., 2009).

It has been demonstrated in the murine models of $S$. Typhimurium infection that it results in the disruption of the indigenous gut microbiota, with a drastic decrease in the total number of bacteria and alteration of the microbiota composition (Barman et al., 2008; Sekirov et al., 2010). In the porcine model of $S$. Typhimurium infection as well, a general reduction of gut microbiota and, in particular, of SCFA-producing bacteria, can be seen (Drumo et al., 2016). Previously, we have confirmed that in humans as well, salmonellosis causes a considerable degree of dysbiosis (Ktsoyan et al., 2015). In the dysbiotic condition accompanied by diarrhea, therefore, the concentration of SCFAs is expected to be decreased in the intestinal milieu, with the corresponding proportional decrease in systemic circulation.

However, the opposite trend was observed, with a significantly increased concentration of SCFAs in the peripheral venous blood of salmonellosis patients (Figure 1). The molar ratio of acetate:propionate:butyrate was also significantly affected, with a drastic decrease of the proportion of acetate and the increased proportions of propionate and butyrate (Table 1). The increase in concentration and proportion of butyrate in peripheral blood may be the consequence of intestinal inflammation and impaired function of colonocytes, for which butyrate is the preferred 


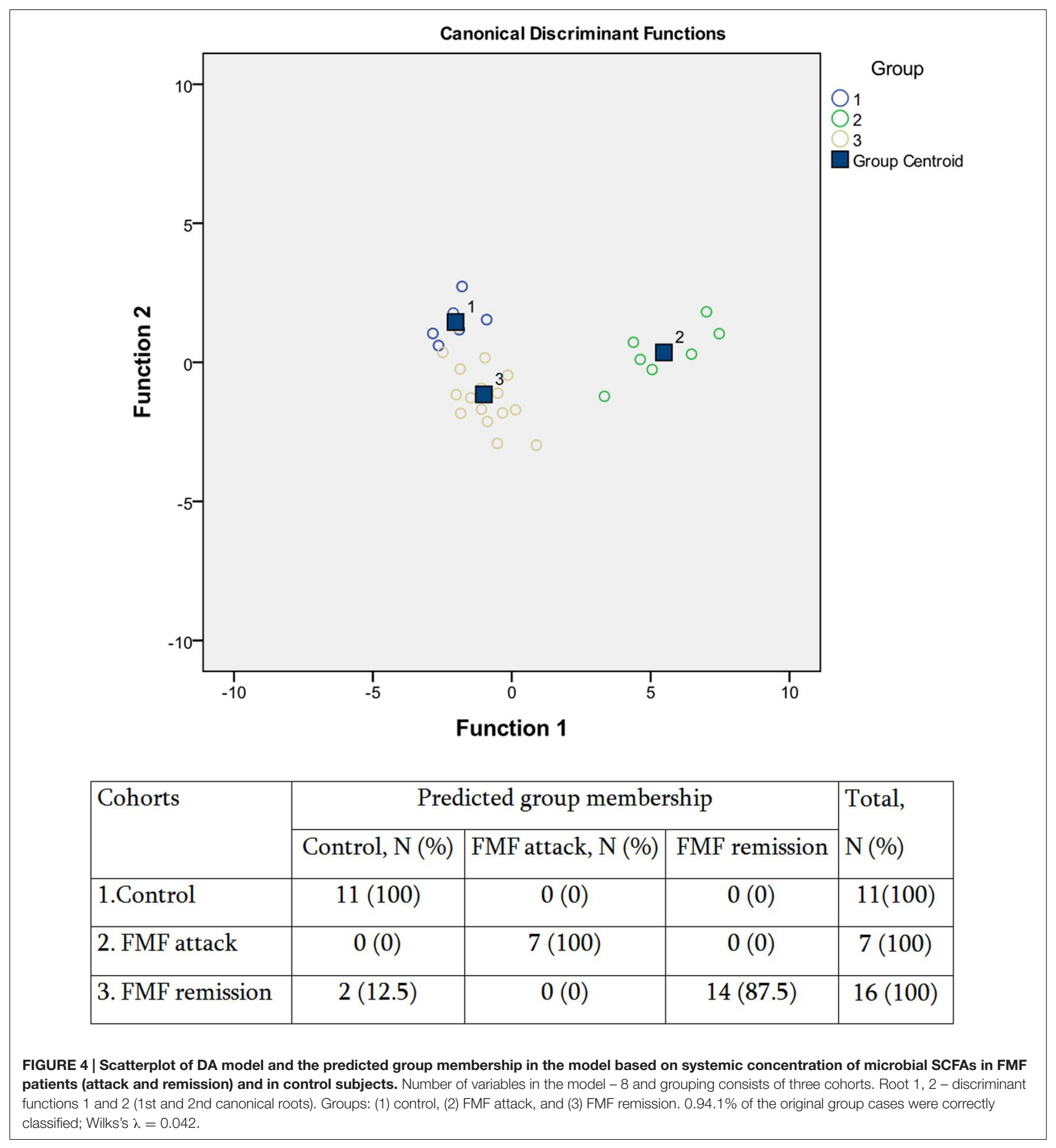

energy source (Velázquez et al., 1997). Thus the less efficient catabolism of butyrate by the inflamed gut tissue may lead to its elevated concentration in peripheral blood.

Propionate is largely metabolized by the liver serving as a precursor for gluconeogenesis (Roy et al., 2006). Severe enterocolitis due to $S$. Enteritidis infection affects liver enzyme levels (González-Quintela et al., 2004) suggesting that the liver function may be impaired in this condition. Because of the increased residual concentration of propionate in blood, the most likely affected function is its transport. In the hepatocytes, organic anion transporters OAT2 and OAT7 are responsible for the transport of propionate and butyrate, correspondingly, from blood, across the sinusoidal membrane (Shin et al., 2007; Islam et al., 2008). The impaired transport from blood to the 
TABLE 1 | Molar ratio of three main systemic SCFAs in salmonellosis and FMF patients.

\begin{tabular}{lccc}
\hline Cohort & Acetate (\%) & Propionate (\%) & Butyrate (\%) \\
\hline Control & $78.19 \pm 37.9$ & $11.49 \pm 4.8$ & $10.32 \pm 3.14$ \\
S. Enteritidis & $35.62 \pm 30.11$ & $50.63 \pm 44.3$ & $13.75 \pm 8.1$ \\
S. Typhimurium & $31.72 \pm 14.5$ & $49.64 \pm 33.1$ & $18.64 \pm 7.0$ \\
FMF (attack) & $87.14 \pm 20.8$ & $7.36 \pm 2.9$ & $5.5 \pm 2.15$ \\
FMF (remission) & $91.15 \pm 32.1$ & $4.925 \pm 2.5$ & $3.925 \pm 3.0$ \\
\hline
\end{tabular}

hepatic tissue may, therefore, explain the insufficient clearance of propionate (and butyrate) by the liver leading to their elevated level in the peripheral blood of the salmonellosis patients. Under the normal circumstances there is a substantial amount of butyrate that leaves the GIT, and it is cleared by the liver (Bloemen et al., 2009). The normal hepatic uptake is capable of clearing even the elevated concentrations of artificially administered butyrate thus preventing the increase in systemic butyrate concentrations (van der Beek et al., 2015).

As mentioned before, acetate is the main SCFA produced by gut microbiota, and there is also a substantial endogenous production of it by the human body (Skutches et al., 1979; Scheppach et al., 1991). Acetate is also involved into a number of anabolic and catabolic biochemical pathways in different tissues and organs (Knowles et al., 1974; Wong et al., 2006; den Besten et al., 2013). It is difficult, therefore, to point out to a single factor that may explain the elevated systemic level of acetate in salmonellosis. Investigation of the uptake of ${ }^{11} \mathrm{C}$-acetate by different normal organs demonstrated that it is absorbed by many organs and, especially actively, by the pancreas, liver, spleen and salivary glands (Song et al., 2009). In the case of salmonellosis many organs can be affected including the liver and spleen. Spadoni et al. (2015) described a gutvascular barrier, which prevents the intestinal bacteria entering the bloodstream and reaching the organs. But the pathogens such as S.Typhimurium can penetrate this barrier employing the pathogenicity island II-encoded type III secretion system and the decreased $\beta$-catenin-dependent signaling in the gut cells. This allows them to enter systemic circulation and reach the liver and spleen thus affecting the function of these organs, possibly including the uptake of acetate. We hypothesize that this scenario may lead to the observed increase of systemic concentration of acetate in salmonellosis patients. The molar ratio of acetate in systemic circulation, however, is substantially lower than that of propionate and, to a lesser extent, of butyrate compared to control (Table 1) thus suggesting that the main organ affected may be the liver where the bulk of propionate is cleared.

Regarding other minor SCFAs, their concentrations in the peripheral blood of salmonellosis patients are also significantly elevated (Figure 1). We cannot, however, compare our results on minor SCFAs with others because of the lack of the relevant publications. In summary, despite that the dysbiosis caused by salmonellosis affects SCFAs-producing bacteria (Drumo et al., 2016) and presumably the gut production of SCFAs as well, the concentrations of SCFAs in the peripheral blood are significantly elevated thus reflecting the failure of disease-affected organs such as the liver to clear SCFAs. This may be also relevant to other metabolites produced by gut microbiota.

In an autoinflammatory condition, FMF, the increase in systemic SCFAs is caused by a different set of factors. First of all, the increase is observed only in the active disease, while it remains close to the control levels in remission except acetate (Figure 3). Previously we have shown that the active FMF is characterized by dysbiosis, with a significant decrease in the total number of gut bacteria and a substantial restructuring of the gut microbiota composition (Khachatryan et al., 2008). In remission, however, these dysbiotic changes are less evident and the diversity values may even exceed that of controls. Thus, in agreement with our earlier observations, in FMF remission we do not see a substantial departure from control in terms of the concentrations of SCFAs in the peripheral blood (Figure 3). The only exception is acetate, the concentration of which is increased almost threefold compared to control. This may be a consequence of subclinical lowgrade inflammation in FMF remission (Manukyan et al., 2008a), which may interfere with the normal and efficient uptake of acetate by different organs (Song et al., 2009). The molar ratio of acetate against two other major SCFAs, propionate and butyrate, is elevated compared to control (Table 1) supporting this notion.

In active FMF, though, the concentrations of almost all SCFAs (except valerate) in the peripheral blood are significantly increased (Figure 3). In this regard the condition resembles the aforementioned infectious disease, salmonellosis. The disease mechanisms, however, are different: FMF is a hereditary disease caused by mutations in the $M E F V$ gene (The French FMF Consortium, 1997; The International FMF Consortium, 1997). Earlier we have suggested that the heightened sensitivity of the immune system conferred by these mutations leads to inappropriate immune responses, launched, in particular, against gut commensals (Manukyan et al., 2008b). The disease exacerbation results in fever and polyserositis, with $95 \%$ of patients experiencing abdominal pain resembling the signs of peritonitis or appendicitis (Livneh and Langevitz, 2000). Despite the different cause of inflammation in FMF attack and salmonellosis, the consequence of both inflammatory conditions is the elevated level of gut-produced SCFAs in systemic circulation (Figures 1 and 3). The enhanced "leak" of microbially produced substances such as hydroxy, branched, cyclopropyl and unsaturated fatty acids, aldehydes, and phenyl derivatives into systemic circulation in FMF has also been noticed by us previously (Ktsoyan et al., 2011, 2013a). Together with the SCFAs data, the presence of various types of microbial products in systemic circulation may suggest a less efficient clearance of these compounds by the liver in these patients. Contrary to salmonellosis, however, the molar ratio of acetate:propionate:butyrate is not significantly affected in FMF patients in remission or attack, although there is a tendency for the increased acetate percentage, with the corresponding decrease in the proportion of propionate and butyrate (Table 1 ). 
In summary, our results suggest that two dissimilar diseases, of infectious and autoinflammatory nature, both result in the elevated level of gut microbiota-produced SCFAs in systemic circulation. Both these disease, however, share a common component, i.e., active inflammation. We hypothesize that the inflammation leads to two major consequences. First, to the compromised gut barrier function (Bischoff et al., 2014), which then results in excessive translation of the luminal content, including SCFAs, into systemic circulation. And second, inflammation affects the intestinal epithelium and the organs beyond the GIT such as the liver that are

\section{REFERENCES}

Ahmer, B. M., and Gunn, J. S. (2011). Interaction of Salmonella spp. with the intestinal microbiota. Front. Microbiol. 6:101. doi: 10.3389/fmicb.2011.00101

Barman, M., Unold, D., Shifley, K., Amir, E., Hung, K., Bos, N., et al. (2008). Enteric salmonellosis disrupts the microbial ecology of the murine gastrointestinal tract. Infect. Immun. 76, 907-915. doi: 10.1128/IAI.01432-07

Bienenstock, J., Kunze, W., and Forsythe, P. (2015). Microbiota and the gut-brain axis. Nutr. Rev. 73, 28-31. doi: 10.1093/nutrit/nuv019

Bischoff, S. C., Barbara, G., Buurman, W., Ockhuizen, T., Schulzke, J. D., Serino, M., et al. (2014). Intestinal permeability - a new target for disease prevention and therapy. BMC Gastroenterol. 14:189. doi: 10.1186/s12876-0140189-7

Bloemen, J. G., Olde Damink, S. W. M., Venema, K., Buurman, W. A., Jalan, R., and Dejong, C. H. C. (2010). Short chain fatty acids exchange: is the cirrhotic, dysfunctional liver still able to clear them? Clin. Nutr. 29, 365-369. doi: 10.1016/j.clnu.2009.10.002

Bloemen, J. G., Venema, K., van de Poll, M. C., Olde Damink, S. W., Buurman, W. A., and Dejong, C. H. (2009). Short chain fatty acids exchange across the gut and liver in humans measured at surgery. Clin. Nutr. 28, 657-661. doi: 10.1016/j.clnu.2009.05.011

Brydges, S., and Kastner, D. L. (2006). The systemic autoinflammatory diseases: inborn errors of the innate immune system. Curr. Top. Microbiol. Immunol. 305, 127-160. doi: 10.1007/3-540-29714-6_7

Byrne, C. S., Chambers, E. S., Morrison, D. J., and Frost, G. (2015). The role of short chain fatty acids in appetite regulation and energy homeostasis. Int. J. Obes. (Lond). 39, 1331-1338. doi: 10.1038/ijo.2015.84

Cerf-Bensussan, N., and Gaboriau-Routhiau, V. (2010). The immune system and the gut microbiota: friends or foes? Nat. Rev. Immunol. 10, 735-744. doi: $10.1038 /$ nri2850

Cook, S. I., and Sellin, J. H. (1998). Review article: short chain fatty acids in health and disease. Aliment. Pharmacol. Ther. 12, 499-507. doi: 10.1046/j.13652036.1998.00337.x

Cummings, J., Pomare, E., Branch, W., Naylor, C., and Macfarlane, G. (1987). Short chain fatty acids in human large intestine, portal, hepatic and venous blood. Gut 28, 1221-1227. doi: 10.1136/gut.28.10.1221

Dankert, J., Zijlstra, J. B., and Wolthers, B. G. (1981). Volatile fatty acids in human peripheral and portal blood: quantitative determination vacuum distillation and gas chromatography. Clin. Chim. Acta 110, 301-307. doi: 10.1016/00098981(81)90359-4

den Besten, G., van Eunen, K., Groen, A. K., Venema, K., Reijngoud, D. J., and Bakker, B. M. (2013). The role of short-chain fatty acids in the interplay between diet, gut microbiota, and host energy metabolism. J. Lipid Res. 54, 2325-2340. doi: 10.1194/jlr.R036012

Drumo, R., Pesciaroli, M., Ruggeri, J., Tarantino, M., Chirullo, B., Pistoia, C., et al. (2016). Salmonella enterica serovar Typhimurium exploits inflammation to modify swine intestinal microbiota. Front. Cell Infect. Microbiol. 5:106. doi: 10.3389/fcimb.2015.00106

González-Quintela, A., Campos, J., Alende, R., López-Soto, A., Tomé, S., Otero, E., et al. (2004). Abnormalities in liver enzyme levels during Salmonella Enteritidis enterocolitis. Rev. Esp. Enferm. Dig. 96, 559-562. doi: 10.4321/S113001082004000800005 involved in metabolism and clearance of microbial metabolites and compounds. These hypotheses, however, need further experimental verification.

\section{AUTHOR CONTRIBUTIONS}

AM and ZG performed sample collection; MZ, KA, AS, and $\mathrm{AH}$ performed experiments; AA and MM performed statistical analyses; RA and ZK performed data analyses, interpretation of data, and writing the paper.

Hawrelak, J. A., and Myers, S. P. (2004). The causes of intestinal dysbiosis: a review. Altern. Med. Rev. 9, 180-197.

Headland, S. E., and Norling, L. V. (2015). The resolution of inflammation: principles and challenges. Semin. Immunol. 27, 149-160. doi: 10.1016/j.smim.2015.03.014

Honda, K., and Littman, D. R. (2012). The microbiome in infectious disease and inflammation. Annu. Rev. Immunol. 30, 759-795. doi: 10.1146/annurevimmunol-020711-074937

Islam, R., Anzai, N., Ahmed, N., Ellapan, B., Jin, C. J., Srivastava, S., et al. (2008). Mouse organic anion transporter 2 (mOat2) mediates the transport of short chain fatty acid propionate. J. Pharmacol. Sci. 106, 525-528. doi: 10.1254/jphs.SC0070291

Khachatryan, Z. A., Ktsoyan, Z. A., Manukyan, G. P., Kelly, D., Ghazaryan, K. A., and Aminov, R. I. (2008). Predominant role of host genetics in controlling the composition of gut microbiota. PLoS ONE 3:e3064. doi: 10.1371/journal.pone.0003064

Kim, C. H., Park, J., and Kim, M. (2014). Gut microbiota-derived short-chain fatty acids, $\mathrm{T}$ cells, and inflammation. Immun. Netw. 14, 277-288. doi: 10.4110/in.2014.14.6.277

Knowles, S. E., Jarrett, I. G., Filsell, O. H., and Ballard, F. J. (1974). Production and utilization of acetate in mammals. Biochem. J. 142, 401-411. doi: 10.1042/bj1420401

Ktsoyan, Z. A., Beloborodova, N. V., Sedrakyan, A. M., Khachatryan, Z. A., Arakelyan, A. A., Aminov, R. I., et al. (2013a). Management of familial Mediterranean fever by colchicine does not normalize the altered profile of microbial long chain fatty acids in the human metabolome. Front. Cell Infect. Microbiol. 3:2. doi: 10.3389/fcimb.2013.00002

Ktsoyan, Z. A., Beloborodova, N. V., Sedrakyan, A. M., Osipov, G. A., Khachatryan, Z. A., Aminov, R. I., et al. (2011). Profiles of microbial fatty acids in the human metabolome are disease-specific. Front. Microbiol. 1:148. doi: 10.3389/fmicb.2010.00148

Ktsoyan, Z. A., Ghazaryan, K., Manukyan, G., Martirosyan, A., Mnatsakanyan, À, Arakelova, K., et al. (2013b). Inflammatory responses to Salmonella infections are serotype-specific. Intern. J. Bacteriol. 2013, 7. doi: 10.1155/2013/168179

Ktsoyan, Z. A., Mkrtchyan, M. S., Zakharyan, M. K., Mnatsakanyan, A. A., Arakelova, K. A., Aminov, R. I., et al. (2015). Differential induction of total IgE by two Salmonella enterica serotypes. Front. Cell. Infect. Microbiol. 5:43. doi: 10.3389/fcimb.2015.00043

Licciardi, P. V., Wong, S.-S., Tang, M. L. K., and Karagiannis, T. C. (2010). Epigenome targeting by probiotic metabolites. Gut Pathog. 2, 24. doi: 10.1186/1757-4749-2-24

Livneh, A., and Langevitz, P. (2000). Diagnostic and treatment concerns in familial Mediterranean fever. Baillieres Best Pract. Res. Clin. Rheumatol. 14, 477-498. doi: 10.1053/berh.2000.0089

Livneh, A., Langevitz, P., Zemer, D., Zaks, N., Kees, S., Lidar, T., et al. (1997). Criteria for the diagnosis of familial Mediterranean fever. Arthritis Rheum. 40, 1879-1885. doi: 10.1002/art.1780401023

Manukyan, G., and Aminov, R. (2016). Update on pyrin functions and mechanisms of familial Mediterranean fever. Front. Microbiol. 7:456. doi: 10.3389/fmicb.2016.00456

Manukyan, G. P., Ghazaryan, K. A., Ktsoyan, Z. A., Tatyan, M. V., Khachatryan, Z. A., Hakobyan, G. S., et al. (2008a). Cytokine profile of Armenian 
patients with familial Mediterranean fever. Clin. Biochem. 41, 920-922. doi: 10.1016/j.clinbiochem.2008.03.017

Manukyan, G. P., Ghazaryan, K. A., Ktsoyan, Z. A., Khachatryan, Z. A., Arakelova, K. A., Kelly, D., et al. (2008b). Elevated systemic antibodies towards commensal gut microbiota in autoinflammatory condition. PLOS ONE 3:e3172. doi: 10.1371/journal.pone.0003172

Nicholson, J. K., Holmes, E., Kinross, J., Burcelin, R., Gibson, G., Jia, W., et al. (2012). Host-gut microbiota metabolic interactions. Science 336, 1262. doi: $10.1126 /$ science. 1223813

Parekh, P. J., Balart, L. A., and Johnson, D. A. (2015). The influence of the gut microbiome on obesity, metabolic syndrome and gastrointestinal disease. Clin. Transl. Gastroenterol. 6, e91. doi: 10.1038/ctg.2015.16

Penders, J., Stobberingh, E. E., van den Brandt, P. A., and Thijs, C. (2007). The role of the intestinal microbiota in the development of atopic disorders. Allergy 62, 1223-1236. doi: 10.1111/j.1398-9995.2007.01462.x

Peters, S. G., Pomare, E. W., and Fisher, C. A. (1992). Portal and peripheral blood short chain fatty acid concentrations after caecal lactulose instillation at surgery. Gut 33, 1249-1252. doi: 10.1136/gut.33.9.1249

Petersen, C., and Round, J. L. (2014). Defining dysbiosis and its influence on host immunity and disease. Cell Microbiol. 16, 1024-1033. doi: 10.1111/cmi. 12308

Pimentel, M., Gunsalus, R. P., Rao, S. S. C., and Zhang, H. (2012). Methanogens in human health and disease. Am. J. Gastroenterol. 1, 28-33. doi: 10.1038/ajgsup

Prakash, S., Rodes, L., Coussa-Charley, M., and Tomaro-Duchesneau, C. (2011). Gut microbiota: next frontier in understanding human health and development of biotherapeutics. Biologics 5, 71-86. doi: 10.2147/BTT.S19099

Roy, C. C., Kien, C. L., Bouthillier, L., and Levy, E. (2006). Short-chain fatty acids: ready for prime time? Nutr. Clin. Pract. 21, 351-366. doi: $10.1177 / 0115426506021004351$

Scheppach, W., Pomare, E. W., Elia, M., and Cummings, J. H. (1991). The contribution of the large intestine to blood acetate in man. Clin. Sci. (Lond.) 80, 177-182. doi: 10.1042/cs0800177

Schuijt, T. J., van der Poll, T., and Wiersinga, W. J. (2012). "Gut microbiome and host defense interactions during critical illness," in Annual Update in Intensive Care and Emergency Medicine, ed. J.-L. Vincent (Berlin: Springer).

Sekirov, I., Gill, N., Jogova, M., Tam, N., Robertson, M., de Llanos, R., et al. (2010). Salmonella SPI-1-mediated neutrophil recruitment during enteric colitis is associated with reduction and alteration in intestinal microbiota. Gut Microbes 1, 30-41. doi: 10.4161/gmic.1.1.10950

Shin, H. J., Anzai, N., Enomoto, A., He, X., Kim, D. K., Endou, H., et al. (2007). Novel liver-specific organic anion transporter OAT7 that operates the exchange of sulfate conjugates for short chain fatty acid butyrate. Hepatology 45, 10461055. doi: 10.1002/hep. 21596

Skutches, C. L., Holroyde, C. P., Myers, R. N., Paul, P., and Reichard, G. A. (1979). Plasma acetate turnover and oxidation. J. Clin. Invest. 64, 708-713. doi: 10.1172/JCI109513

Song, W. S., Nielson, B. R., Banks, K. P., and Bradley, Y. C. (2009). Normal organ standard uptake values in carbon-11 acetate PET imaging. Nucl. Med. Commun. 30, 462-465. doi: 10.1097/MNM.0b013e32832aa7ce
Spadoni, I., Zagato, E., Bertocchi, A., Paolinelli, R., Hot, E., Di Sabatino, A., et al. (2015). A gut-vascular barrier controls the systemic dissemination of bacteria. Science 350, 830-834. doi: 10.1126/science.aad0135

Stecher, B., Chaffron, S., Käppeli, R., Hapfelmeier, S., Freedrich, S., Weber, T. C., et al. (2010). Like will to like: abundances of closely related species can predict susceptibility to intestinal colonization by pathogenic and commensal bacteria. PLoS Pathog. 6:e1000711. doi: 10.1371/journal.ppat.10 00711

Sunkara, L. T., Achanta, M., Schreiber, N. B., Bommineni, Y. R., Dai, G., Jiang, W. et al. (2011). Butyrate enhances disease resistance of chickens by inducing antimicrobial host defense peptide gene expression. PLOS ONE 6:e27225. doi: 10.1371/journal.pone.0027225

Sunkara, L. T., Jiang, W., and Zhang, G. (2012). Modulation of antimicrobial host defense peptide gene expression by free fatty acids. PLOS ONE 7:e49558. doi: 10.1371/journal.pone.0049558

The French Fmf Consortium (1997). A candidate gene for familial Mediterranean fever. Nat. Genet. 17, 25-31. doi: 10.1038/ng0997-25

The International Fmf Consortium (1997). Ancient missense mutations in a new member of the RoRet gene family are likely to cause familial Mediterranean fever. Cell 90, 797-807. doi: 10.1016/S0092-8674(00)80539-5

van der Beek, C. M., Bloemen, J. G., van den Broek, M. A., Lenaerts, K., Venema, K., Buurman, W. A., et al. (2015). Hepatic uptake of rectally administered butyrate prevents an increase in systemic butyrate concentrations in humans. J. Nutr. 145, 2019-2024. doi: 10.3945/jn.115.211193

Velázquez, O. C., Lederer, H. M., and Rombeau, J. L. (1997). Butyrate and the colonocyte. Production, absorption, metabolism, and therapeutic implications. Adv. Exp. Med. Biol. 427, 123-134.

Vinolo, M. A. R., Rodrigues, H. G., Nachbar, R. T., and Curi, R. (2011). Regulation of Inflammation by short chain fatty acids. Nutrients 3, 858-876. doi: $10.3390 /$ nu3100858

Winter, S. E., Thiennimitr, P., Winter, M. G., Butler, B. P., Huseby, D. L., Crawford, R. W., et al. (2010). Gut inflammation provides a respiratory electron acceptor for Salmonella. Nature 467, 426-429. doi: 10.1038/nature09415

Wong, J. M., de Souza, R., Kendall, C. W., Emam, A., and Jenkins, D. J. (2006). Colonic health: fermentation and short chain fatty acids. J. Clin. Gastroenterol. 40, 235-243. doi: 10.1097/00004836-200603000-00015

Conflict of Interest Statement: The authors declare that the research was conducted in the absence of any commercial or financial relationships that could be construed as a potential conflict of interest.

Copyright (c) 2016 Ktsoyan, Mkrtchyan, Zakharyan, Mnatsakanyan, Arakelova, Gevorgyan, Sedrakyan, Hovhannisyan, Arakelyan and Aminov. This is an openaccess article distributed under the terms of the Creative Commons Attribution License (CC BY). The use, distribution or reproduction in other forums is permitted, provided the original author(s) or licensor are credited and that the original publication in this journal is cited, in accordance with accepted academic practice. No use, distribution or reproduction is permitted which does not comply with these terms. 